\title{
TESTING, SIMULATING AND UNDERSTANDING UNDER-PLATFORM DAMPER DYNAMICS
}

\author{
C. Gastaldi, Muzio M. Gola \\ Politecnico di Torino \\ Corso Duca degli Abruzzi 24, Torino, Italy \\ e-mail: \{chiara.gastaldi,muzio.gola\}@polito.it
}

Keywords: under-platform damper, friction, damping, contact parameters

Abstract. Underplatform dampers (UPDs) are widely used as a source of friction damping and are frequently incorporated into compressors and turbines for both aircraft and powerplant applications to mitigate the effects of resonant vibrations on fatigue failure.

The need for reliable models of UPDs has led to a considerable amount of literature in the last three decades. The standard approach is to fine-tune and experimentally validate the UPDs models by comparing measured and calculated vibration response of blade pairs. With this approach one cannot investigate the damper behaviour directly and no measurements of the contact parameters can be undertaken. The experimental-numerical method proposed by these authors overcomes this problem through the combined use of purposely developed tools:

- a test rig capable of measuring directly the damper working parameters (i.e. damper hysteresis cycle and kinematics);

- error estimates of measured and derived forces and displacements ;

- a numerical routine representing the damper between the platforms solved either in the time or in the frequency domain;

- a robust procedure for the estimation of friction and stiffness parameters at the contact from experimental data.

These tools combined together offer concrete prospects of success in damper simulation and design. On one hand, they allow mapping the values of contact parameters under different working conditions (i.e. frequency, centrifugal load and presencelabsence of damper rolling), thus ensuring valuable predictions both at the design and at the simulation stage. On the other hand the experimental-numerical method offers a clear understanding of all contact events (stick, slip, lift) which take place during the cycle, and on how they influence the damping performance. This deeper insight is the basis to actual design and optimization criteria.

Purpose of the paper is to illustrate the tools and methods through the analysis of a family of rigid bar dampers with a curved-flat cross section. A series of diagrams fit to describe the damper behaviour will be presented and used to estimate relevant friction contact parameters. 


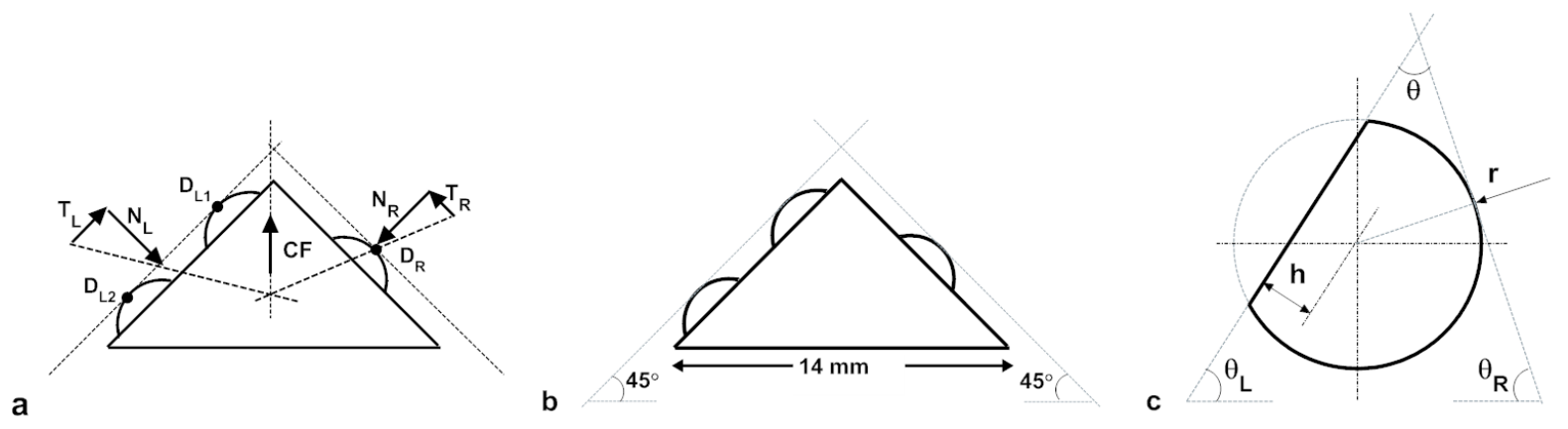

Figure 1: Laboratory three-point damper a- IP hysteresis cycle b- Tangential/Normal force ratio during the cycle c- Contact Forces Diagram

\section{INTRODUCTION}

The dedicated damper routine developed by the AERMEC group [1, 2, 3] combines numerical simulation with a trustworthy experimental approach for these reasons:

- experimental observations can be used as a benchmark to draw the appropriate values of contact parameters (local friction coefficients and contact stiffness) to be used as input to a numerical model which represents the dynamics of the damper between the two platforms.

- the validated routine becomes a design and optmization tool.

The outcome of the design and optimization tool are highly dependent on friction interface parameters, as demonstrated in [4]. Trustworthy predictions cannot prescind from the experimental investigation of friction interface parameters, and studies on the measurement of friction coefficients and contact stiffness values appear in the literature to this day [5].

The first part of the paper will illustrate the test rig and the numerical model applied to a threepoint laboratory damper shown in Fig. 1a-b. It will be shown how experimental data can be used to understand the damper behaviour and then to estimate friction contact parameters. Moreover, it will be highlighted how the simplified configuration of the three-point laboratory damper (three equal non-conforming contacts with known positions) makes the tuning process easier.

The second part of the paper will instead focus on two real optimized curved-flat dampers, whose configurations (i.e. angles and depth of cut) were chosen following the pre-optimization technique presented in [6]. The purpose of the paper is to highlight the additional effort necessary to correctly model differenct contact interfaces (both conforming and non-conforming) and to propose a robust data-processing technique to estimate contact parameters.

\section{THE TEST RIG}

The experimental investigation here summarized takes advantage of a test rig (Fig.2) purposely developed at the AERMEC lab [1] representing a damper between two platforms.

The damper is loaded by a deadweight simulating the centrifugal force. The cable holes are properly oriented and pass through the damper center of mass: their sections shrink down to match the cables' diameter in correspondence of the center of mass to exactly reproduce the loading conditions encountered on a bladed disk. 

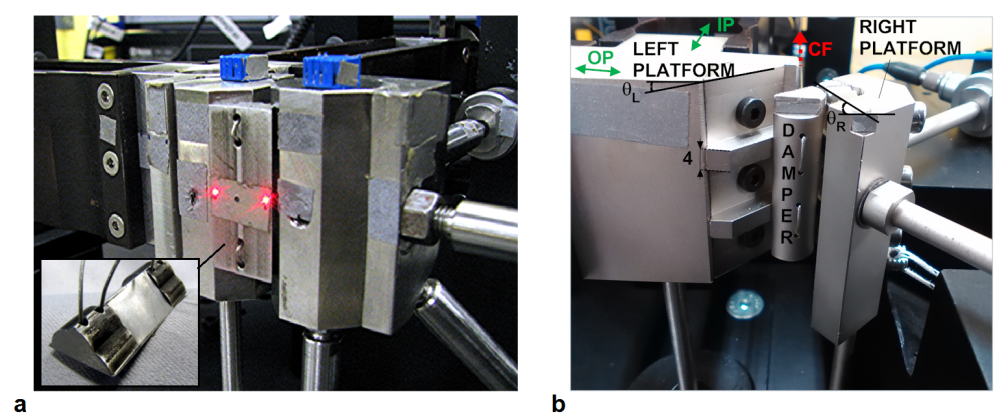

Figure 2: Experimental setup featuring a- the three point laboratory damper and b- a curved-flat damper

Table 1: Uncertainty on experimental data

\begin{tabular}{cc}
\hline Quantity & Standard deviation \\
\hline Right contact force $\left(N_{R}, T_{R}\right)$ & $3 \%(0.5 \mathrm{~N})$ \\
\hline Left contact force $\left(N_{L}, T_{L}\right)$ & $3-5 \%(0.7-0.9 \mathrm{~N})$ \\
\hline Left c. force application point & $0.5 \mathrm{~mm}$ \\
\hline Platforms relative movement $\left(w_{L P}-w_{R P}\right)$ & $0.08 \mu \mathrm{m}$ \\
\hline Rotation $(\beta)$ & $5 \%\left(0.6 \cdot 10^{-4} \mathrm{rad}\right)$ \\
\hline
\end{tabular}

The left blade platform, which serves as input motion to the system, is connected to two perpendicular piezoelectric actuators. The actuators are capable of reproducing any in-plane periodic displacement, however the cases analyzed in this paper all reproduce the typical blades' InPhase (IP) motion. The right blade platform, connected, by means of a tripod, to two force sensors measures the contact forces transmitted between the platforms, through the damper. A laser head measures the platforms' relative displacement and damper kinematics.

The forces transmitted between the platforms through the damper are measured and related to the measured relative platform movement in order to record the damper hysteresis loop (e.g shown in Fig.3).

A complete description of the test rig components, calibration procedures and the error estimation techniques can be found in [1,7]. Table 1 lists all the measured and derived quantities together with their level of uncertainty. These results led the authors to believe that the accuracy of force and kinematic measurements offers a trustworthy basis on which to build the contact parameter estimation technique.

\section{UNDERSTANDING AND SIMULATING A LABORATORY THREE-POINT DAMPER}

\subsection{Relevant Diagrams}

The hysteresis cycle in Fig.3a plots the force (vertical componentin the I-P case) transmitted to the right platform plotted as a function of the corresponding component of the relative displacement between platforms. Reference points on the hysteresis loop have been marked by a symbol and a number, repeated on the corresponding points on other diagrams: they are useful to guide the analysis of the cycle by cross-comparison.

For each hysteresis cycle (Fig.3a) a set of experimental diagrams can be produced to investigate the damper behavior. Numerical results (dashed line) are shown together with their experimental match (solid line). The experimental results allow to validate the numerical model (by 

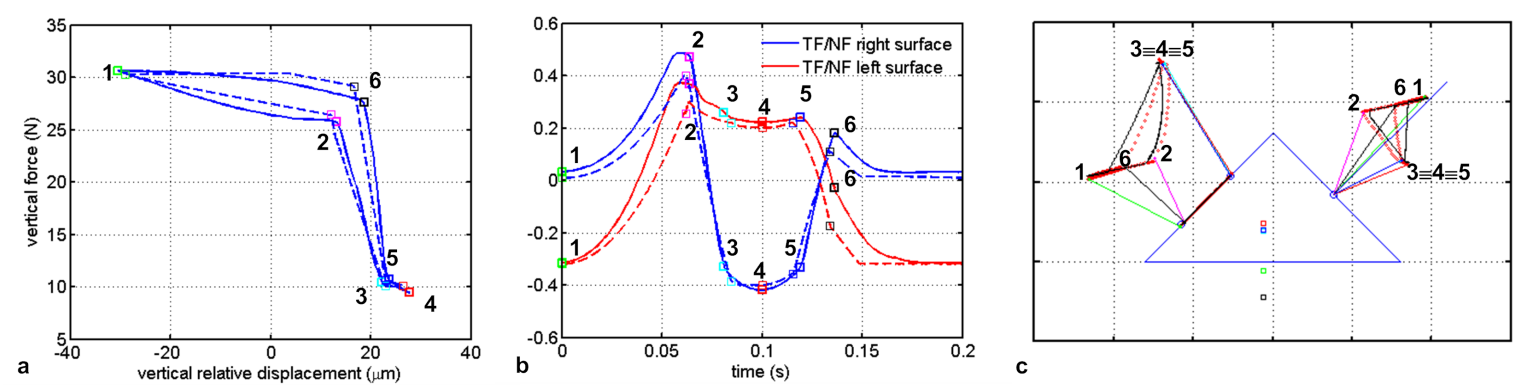

Figure 3: Laboratory three-point damper a- IP hysteresis cycle b- Tangential/Normal force ratio during the cycle c- Contact Forces Diagram
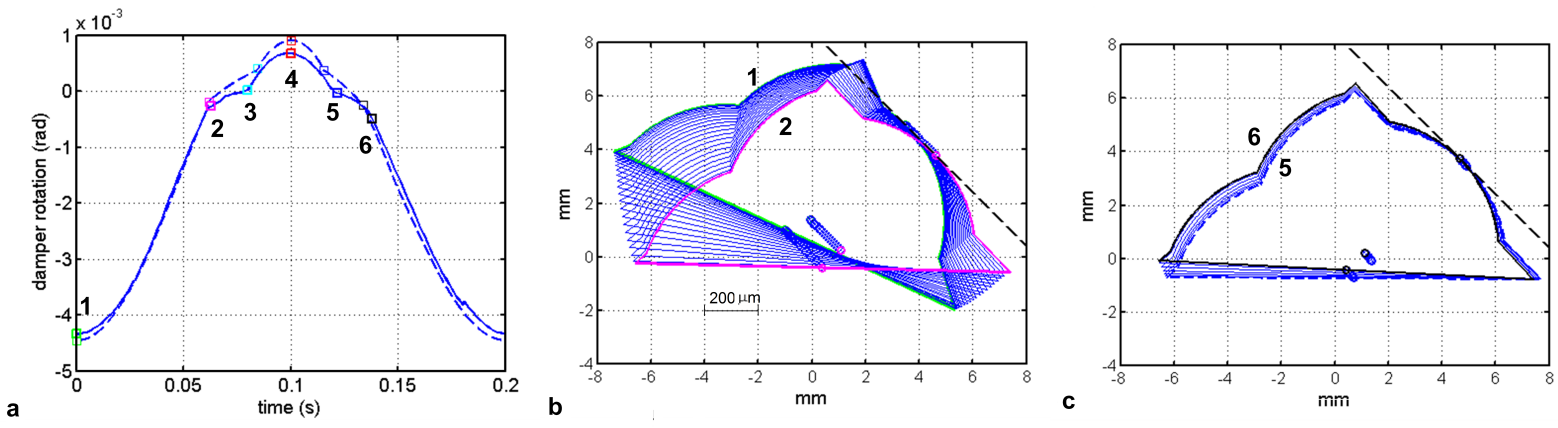

Figure 4: Laboratory three-point damper a- Rotation against time. b- Kinematic reconstruction from marker 1 to 2 . c- Kinematic reconstruction from marker 5 to 6

comparing the two sets of results) and to estimate the contact parameters used to obtain the simulated cycle. These diagrams are:

- T/N force ratios (Fig. 3b) : it represents the ratio of the total tangential and normal force components on the left and right contact surfaces plotted as a function of time. The flat portions of each line may indicate a slip phase subject to cross-confirmation by other diagrams - on an interface: in such case the ratio $\mathrm{T} / \mathrm{N}$ will represent a friction coefficient.

- Contact forces diagram (Fig. 3c): it represents -) the vectors of forces transmitted between the platforms -) the damper surfaces and their points of application.

- Damper rotation (b) against time (Fig. 4a).

- Damper kinematic reconstruction (i.e. Fig. 4b-c): a visual representation of damper positions with magnified (x 100) displacements and rotation.

\subsection{The damper numerical routine}

The interpretation of experimental results is quite complex because it requires to relate the behaviour of forces and motions in order to assess the working mode in each part of the hysteresis cycle. A numerical model is then necessary to analyze each tract of the hysteresis cycle by precisely identifying stick or slip conditions and the related exchanged forces, as shown in 

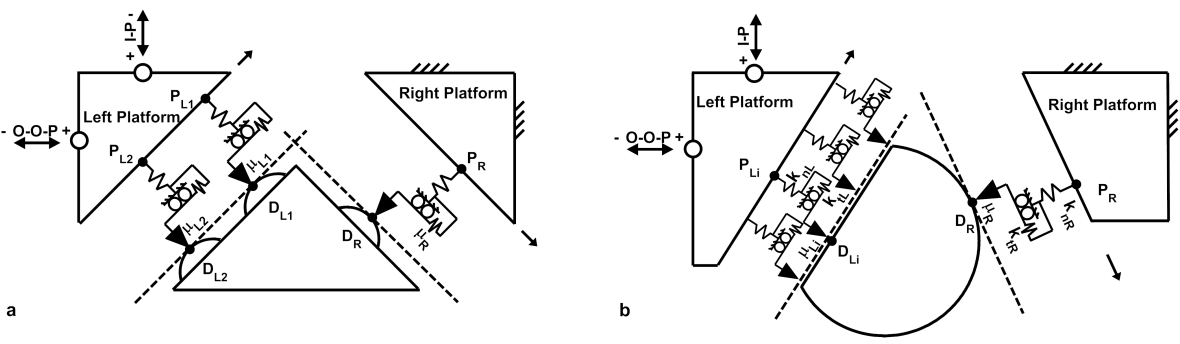

Figure 5: Damper numerical routine featuring a Laboratory damper b Curved-flat damper

Table 2: Step by step analysis of the cycle in Fig. 3-4

\begin{tabular}{c||c|c|c|c||c|c}
\hline Stage & $T_{L} / N_{L}$ & $N_{L}$ position & $D_{L 1}$ & $D_{L 2}$ & $T_{R} / N_{R}$ & $D_{R}$ \\
\hline $1-2$ & Varying & on $D_{L 2}$ & Separation & Stick & Varying & Stick \\
\hline 2-3 & $\approx$ Constant & b/w $D_{L 1}$ and $D_{L 2}$ & Slip $_{+}$ & Slip & Varying & Stick \\
\hline 3-4 & Constant & on $D_{L 1}$ & Slip $_{+}$ & Separation & Constant & Slip \\
\hline 4-5 & Constant & on $D_{L 1}$ & Slip $_{+}$ & Separation & Varying & Stick \\
\hline $5-6$ & Varying & b/w $D_{L 1}$ and $D_{L 2}$ & Slip $_{+}$ & Stick & Varying & Stick \\
\hline 6-1 & Constant & on $D_{L 2}$ & Separation & Slip & Varying & Stick \\
\hline
\end{tabular}

Table 2. A functional scheme of the numerical model adapted to both damper configurations is shown in Fig. 5. All contact interfaces are modeled using an Iwan-type contact model which requires three contact parameters: normal and tangential contact stiffness and friction coefficient. A complete description of the damper numerical routine, which can be solved either in the time or in the frequency domain, can be found in [1] and [2].

\subsection{Estimation of contact parameters}

\subsubsection{Friction coefficients}

Friction coefficients are estimated using the $\mathrm{T} / \mathrm{N}$ force ratios (Fig. 3b). The right one poses no problem since there is only one contact point on that side of the damper. The left surface is, on the other hand, the result of the combination of the two contact points. Initially $\mu_{L 1}=\mu_{L 2}$ is assumed, and by considering in addition another tract where only one of the left contact points is actually in contact and is slipping (e.g. stage 3-4 for $\mu_{L 1}$ and stage 6-1 for $\mu_{L 2}$ referring to Fig. 3b-c) $\mu_{L 1}$ and $\mu_{L 2}$ can be determined. The resulting values of friction coefficients are in this case : $\mu_{L 1}=0.22, \mu_{L 2}=0.34$ and $\mu_{R}=0.4$.

\subsubsection{Contact stiffness values}

Since all contact points share the same geometry it is here assumed that they all share the same normal and tangential stiffness values $k_{n}$ and $k_{t}$. The normal contact stiffness $k_{n}$ was estimated using the normal displacement-normal force curve of a cylinder pressed against a plane[9]-[10].

The tangential contact stiffness was estimated using the full-stick (all contact points in contact and in stick) cycle slope of the OP hysteresis cycles (here not shown)[1]. It shold be noted that this slope is the same in all investigated cases and does not change when the platform starts closing. This due to the fact that during that stage all contact points are repeatably in the same 
contact condition. The cycle slope is a composite effect of normal and tangential stiffness values at all contacts. The resulting values of contact stiffness are in this case : $k_{n}=2.4 \mathrm{~N} / \mu \mathrm{m}$, $k_{t}=1.6 \mathrm{~N} / \mu \mathrm{m}$.

\section{LIMITS OF THE CURRENT PRACTICE ON LABORATORY UPDS INVESTIGA- TION}

The methods developed for the three-point laboratory damper are successful in exploring and understanding its behaviour, however several shortcomings still remain to be tackled.

1. The laboratory damper here analyzed is a non-optimized configuration (i.e. large rolling motion caused by contact point lift-off minimizes the dissipated energy per cycle).

2. In order for the design tool to be effective, its simulation capabilities have to include dampers with other geometries (i.e. curved-flat damper as shown in Fig.1)

These authors have tackled point 1 in [6], by proposing a pre-optimization method for curvedflat UPDs. This method was successful in identifying and excluding those damper configurations (choice of $\theta_{L}, \theta_{R}$ and $h$ shown in Fig. 1c) leading to undesirable damper behaviour (contact point lift-off, jamming etc.).

Concerning point 2, a few successful attempts to model curved-flat UPDs have been presented by these authors $[2, ?]$. However some of the assumptions made for the three-point laboratory damper are not valid for other damper geometries, for instance:

- number of contact points on the flat side;

- position of the contact points on the flat side;

- contact stiffness values of the flat side are potentially different from those representing the cylindrical contact point.

These differences make the tuning procedure described in 3.3 not completely suitable when estimating contact parameters relative to the curved-flat dampers-platforms interface. The purpose of this paper is to overcome these shortcomings by proposing a new method with a solid experimental basis and a fine data processing technique.

\section{EXPLORING CURVED-FLAT DAMPERS}

\subsection{Selected configurations}

As mentioned before the selected damper configurations are the result of a pre-optimization process which aims at maximizing the dissipated energy [6]. The parameters which influence the damper behaviour are (see Fig.1):

- the non-dimensional "residual radius" $h / r$;

- the angles of the platforms $\theta_{R}$ and $\theta_{L}$.

- the value of the friction coefficients $\mu_{R}$ and $\mu_{L}$;

The selected damper configurations are:

- A: $\theta_{L}=55^{0}, \theta_{R}=65^{0}, h / 2=0.2$ 

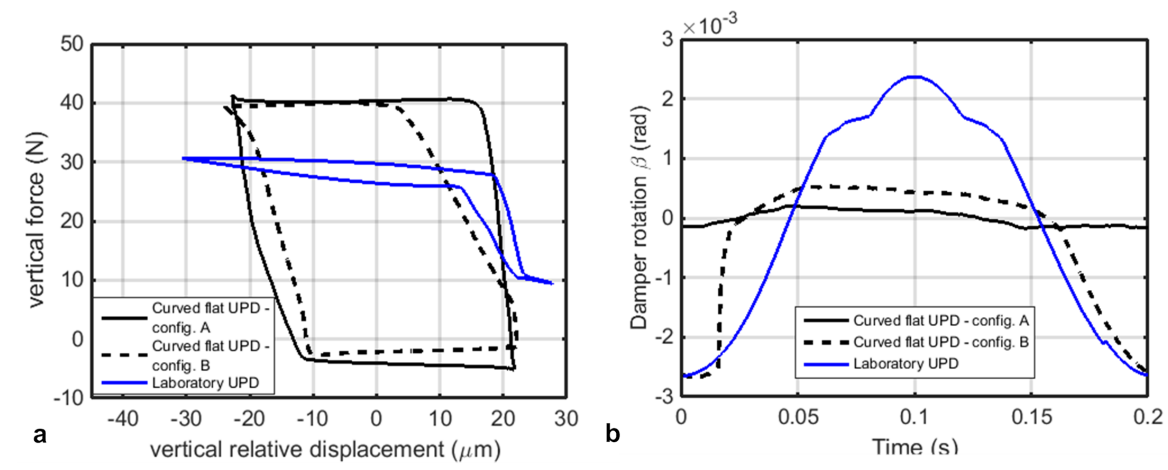

Figure 6: a Hysteresis cycles and b Damper rotation signals for an applied centrifugal load of $C F=4.65 \mathrm{~kg}$ and a $5 \mathrm{~Hz} \pm 30 \mu m$ IP imposed displacement

- B: $\theta_{L}=35^{0}, \theta_{R}=65^{0}, h / 2=0.2$

While $h / r, \theta_{L}$ and $\theta_{R}$ are parameters the designer can act upon, friction coefficients are unknown constraints measured a-posteriori.

Figure 6 shows the hysteresis cycles and the rotation signals for the two curved-flat damper configurations and for the above mentioned laboratory damper. The boundary conditions imposed to the dampers (i.e. centrifugal load, imposed displacement and frequency) are the same for all three cases. The results shown here were produced by a $5 \mathrm{~Hz}$ excitation because the signals are cleaner and easier to manipulate. However, it should be noted that contact stiffness values did not change for all investigated frequencies (i.e. up to $160 \mathrm{~Hz}$ ).

Severe differences are noted among the three configurations:

- force levels: the curved-flat damper which display a set of friction coefficients $\left(\mu_{R}=\right.$ $\left.0.65, \mu_{L}=0.5\right)$ and $\left(\mu_{R}=0.78, \mu_{L}=0.5\right)$ for configurations A and B respectively, ensure slip force levels higher than those encountered for the laboratory damper $\left(\mu_{R}=\right.$ $\left.0.4, \mu_{L} \approx 0.3\right)$.

- Configuration A (i.e. choice of angles and parameter $h / r$ ) manages to avoid lift-off completely, as shown by the rotation signal in Fig. 6, and thus to maximize the dissipated energy.

- Configuration B does encouter some lift-off and therefore a larger rotation, it is however a significant improvement if compared to the laboratory damper.

\section{CONTACT PARAMETERS ESTIMATION FOR CURVED-FLAT DAMPERS}

Both the pre-optimization technique presented in [6] and the damper numerical routine itself need a robust model of contact interfaces. The purpose of this section is to present the new configuration introduced in the numerical routine to model a curved-flat damper and the new experimental capabilities developed at AERMEC to estimate the relevant contact parameters. This section will focus on the contact point position and on the contact stiffness values. The technique to estimate friction coefficients developed for the three-point laboratory damper can be applied without further modifications to any other damper configuration. 

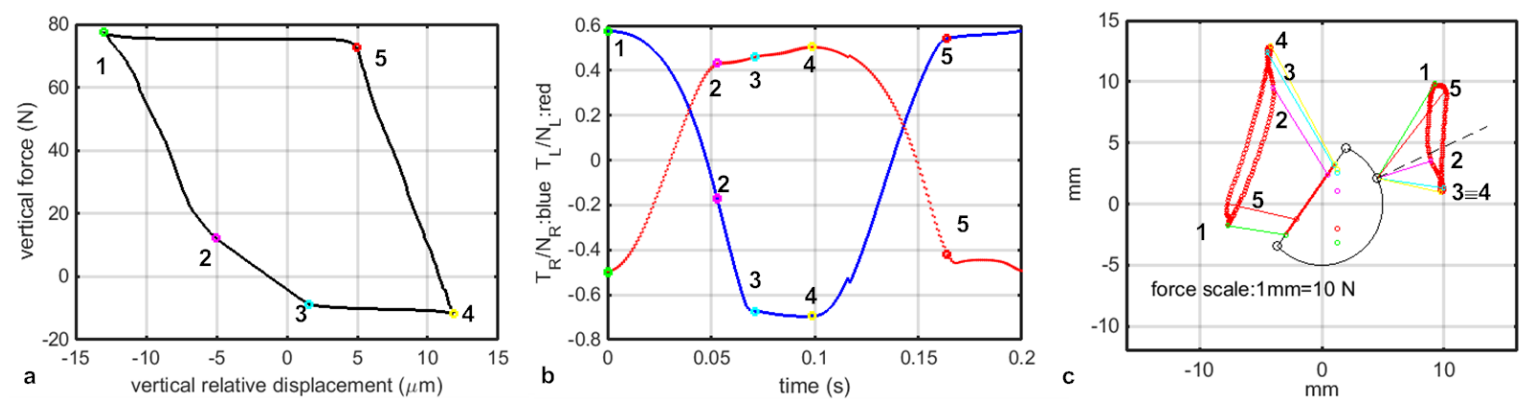

Figure 7: Curved-flat damper a- IP hysteresis cycle b- Tangential/Normal force ratio during the cycle c- Contact Forces Diagram

\subsection{Contact points number and position}

While the number and position of contact points used to model the laboratory UPD (see Fig. 5a) was determined by the damper configuration itself, while the flat portion of the curved-flat damper requires additional attention. A few successful attempts to model a curved-flat UPD using only two contact points on the left side have been carried out in the past [2]. However, without additional experimental evidence the system is under-determined and selecting the position of the contact points and the contact stiffness values requires a cumbersome case-by-case fine-tuning procedure. Section 6.1.2 proposes a novel method to estimate the normal contact stiffness per unit length. Therefore it is sensible to envision a series of contact points uniformly distributed over the contact interface, the higher the number of contact points, the finer the representation of the interface.

\subsubsection{Contact stiffness values}

As mentioned before the contact stiffness tuning technique developed for the laboratory damper is not valid anymore. While the normal contact stiffness on the right (i.e. cylindrical) side can still be deduced using the normal displacement-normal force curve of a cylinder pressed against a plane[9]-[10], the remaining unknowns $\left(k_{t L}, k_{t R}\right.$ and $\left.k_{n L}\right)$ need additional information which can only be provided by a robust experimental basis.

\subsubsection{Normal contact stiffness at the flat interface}

Referring to Fig. 8 it is possible to write the expression of the rotation $\beta$ at a given instant in time as:

$$
\beta=\frac{n(x)}{x-\left(\frac{L}{2}-3 a\right)}
$$

where $n(x)$ is the displacement in the normal direction at a given position $\mathrm{x}$ of the flat interface. The reference system $\mathrm{x}$ is parallel to the contact and its origin lies in the middle of the flat interface. Let us divide the flat interface into $\mathrm{k}$ segments each $\Delta x$ long. If the normal stiffness is uniformly distributed the normal stiffness pertaining to one of these segments is:

$$
k_{n i}=\frac{d k_{n L}}{d x} \cdot \Delta x \quad 1 \leq i \leq k
$$


If the force per unit length $\mathrm{q}(\mathrm{x})$ is known, e.g. $q(x)=\frac{q_{\max }}{3 a} \cdot\left(x-\left(\frac{L}{2}-3 a\right)\right)$, it is possible to substitute the expression of $n(x)$ inside Eq. 1.

$$
\beta=\frac{q(x) \cdot \Delta x}{\frac{d k_{n L}}{d x} \Delta x \cdot\left(x-\left(\frac{L}{2}-3 a\right)\right)}
$$

where $a$ represents the distance between $N_{L}$ application point and the nearest flat surface edge, as shown in Fig. 8b. If the force is uniformly distributed $q(x)$ will act on a portion of flat surface 3a long.

It is possible to express $q(x)$ as a function of the moment $M$ produced by the resultant $N_{L}$ around the origin shown in Fig. 8a.

$$
\begin{aligned}
M & =\int_{\frac{L}{2}-3 a}^{\frac{L}{2}} \frac{q_{\max }}{3 a}\left(x-\left(\frac{L}{2}-3 a\right)\right) \cdot x \cdot d x \\
& =\frac{3}{2} q_{\max } \cdot a \cdot\left(\frac{L}{2}-a\right)
\end{aligned}
$$

Substituting Equ. 4 in Equ. 3, it is possible to express $\frac{d k_{n L}}{d x}$ as:

$$
\begin{aligned}
\frac{d k_{n L}}{d x} & =\frac{q(x)}{\beta} \\
& =\frac{2 M}{9 a^{2} \cdot\left(\frac{L}{2}-a\right)}
\end{aligned}
$$

Figure 8c plots $2 \cdot M$ against $9 a^{2} \cdot\left(\frac{L}{2}-a\right)$ for the case shown in Fig. 7. The slope highlighted in the figure is the normal contact stiffness per unit length. The shape is slightly distorted:

- for low values of rotation/moment: this is due to the fact that the assumption made in Equ. 3 (i.e. that rotation and moment signals in time are synchronous) is not perfectly true due to minor distorsions caused by spurious movements of the left platform;

- close to markers 1/5: during those stages the left contact force resultant $N_{L}$ application point is close to the lower edge, a situation similar to lift-off where the length of $a->0$

The resulting values of $\frac{d k_{n L}}{d x}$ for both configurations of curved-flat UPDs are reported in Table 6.1.2. The results have been repeated on both tracts (i.e. ascending and descending) of the shape in Fig. 8c and repeated using repeated independent measurements.

\subsubsection{Tangential Contact Stiffness values}

The tangential contact stiffness values are derived directly from experimental data. The available laser heads are pointed on damper and platform protrusions purposely machined to be perpendicular to the contact surfaces, as shown in Fig. 9a.

The laser signal is then plotted against the corresponding tangential force ( $T_{R}$ or $T_{L}$ respectively), as shown in Fig. $9 \mathrm{~b}$ and $\mathrm{c}$ for the curved-flat damper (configuration A). It should be noted that the tangential force on the cylindrical side $T_{R}$ is measured using the load cells, while the tangential force resultant on the flat side $T_{L}$ is reconstructed using the procedure described 
Table 3: Flat interface normal contact stiffness per unit length

\begin{tabular}{c||c|c|c|c}
\hline Damper configuration & Centrifugal Load & Repetition & $\frac{d k_{n L}}{d x}\left(\mathrm{~N} / \mathrm{mm}^{2}\right)$ & $\sigma\left(\mathrm{N} / \mathrm{mm}^{2}\right)$ \\
\hline $\mathrm{A}$ & $4.65 \mathrm{~kg}$ & 1 & 18900 & 1387 \\
\hline $\mathrm{A}$ & $4.65 \mathrm{~kg}$ & 2 & 19004 & 1831 \\
\hline $\mathrm{A}$ & $8.65 \mathrm{~kg}$ & 1 & 18054 & 847 \\
\hline $\mathrm{A}$ & $8.65 \mathrm{~kg}$ & 2 & 19393 & 669 \\
\hline \hline $\mathrm{B}$ & $4.65 \mathrm{~kg}$ & 1 & 1334 & 362 \\
\hline $\mathrm{B}$ & $4.65 \mathrm{~kg}$ & 2 & 1231 & 79 \\
\hline $\mathrm{B}$ & $8.65 \mathrm{~kg}$ & 1 & 6412 & 558 \\
\hline $\mathrm{B}$ & $8.65 \mathrm{~kg}$ & 2 & 6586 & 581 \\
\hline \hline
\end{tabular}
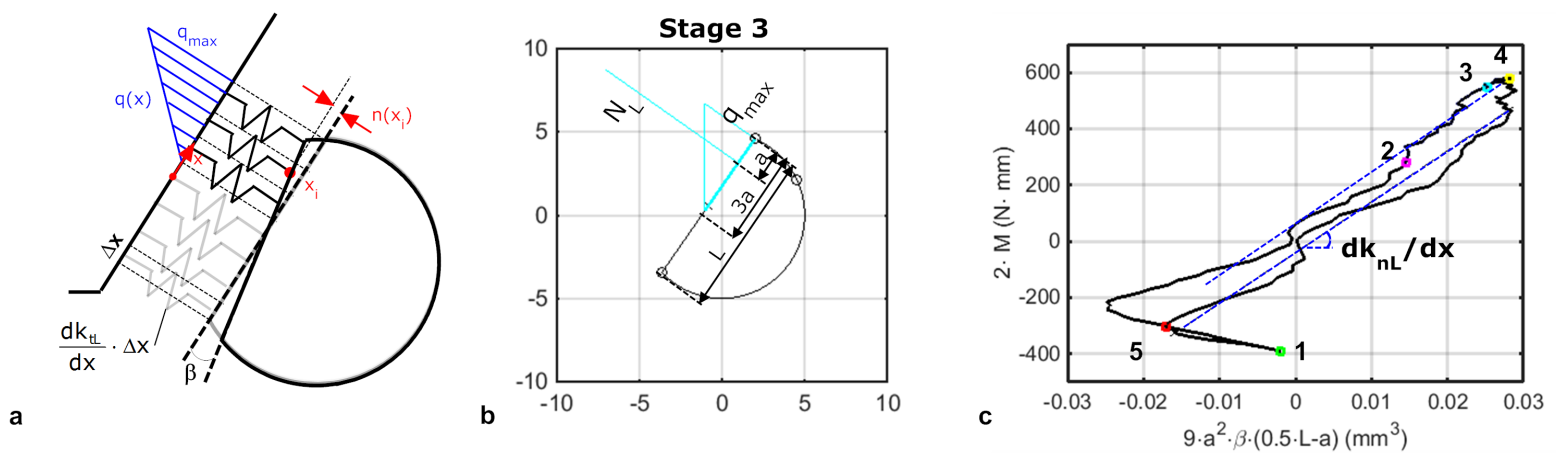

Figure 8: a- Scheme representing the flat surface model. b- Force component $N_{L}$ and corresponding force per unit-length on the flat surface at marker 3 for the cycle in Fig. 7a. c- Flat interface normal contact stiffness per unit length relative to the cycle shown in Fig. 7.
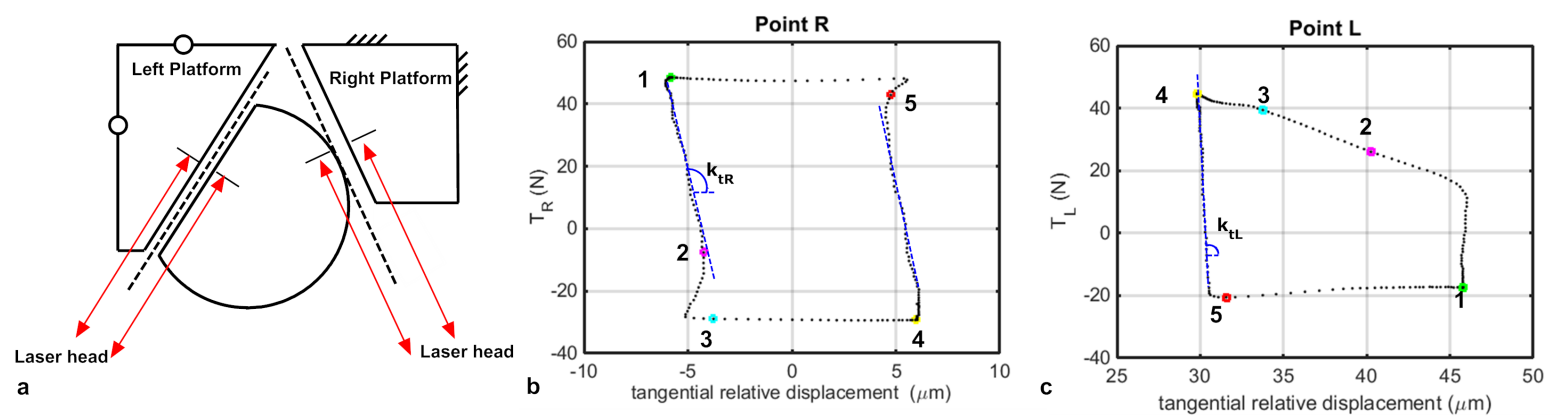

Figure 9: a- Functional scheme representing the experimental procedure used to estimate the tangential contact stiffness values b- Hysteresis at the right contact c- Hysteresis at the left interface 

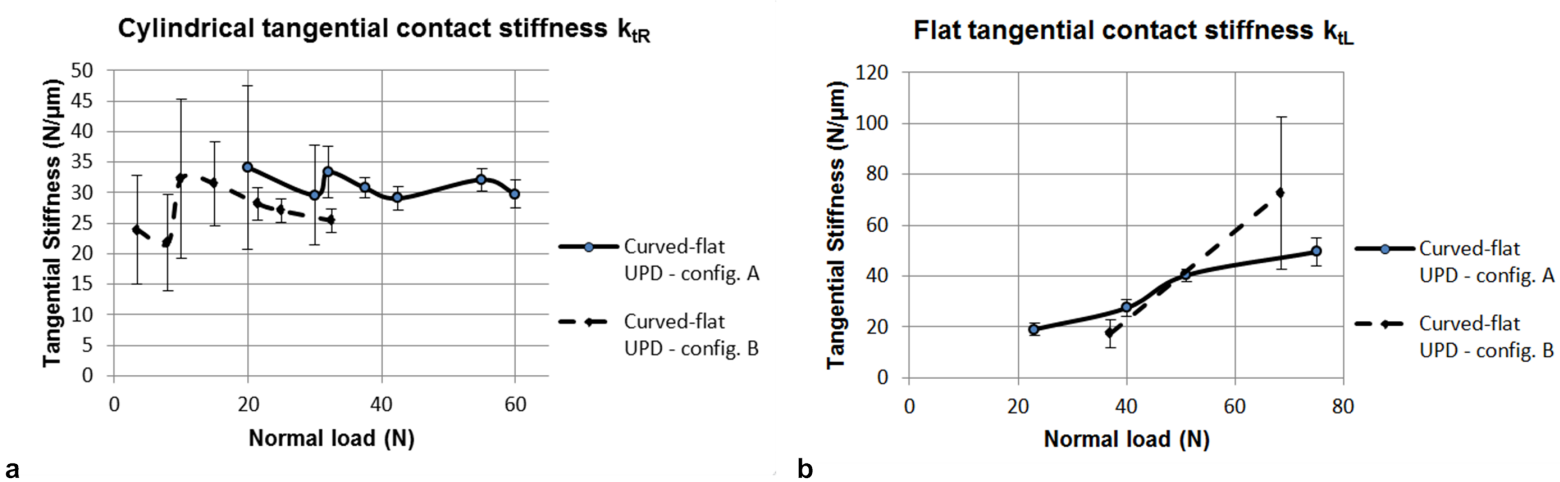

Figure 10: Curved-flat damper a- IP hysteresis cycle b- Tangential/Normal force ratio during the cycle c- Contact Forces Diagram

in Fig.1a.

For each contact interface the stages corresponding to a stick state are identified using the Tangential/Normal force ratio diagram (i.e. in Fig. 7b). The stages are "1-3" and "4-5" for the right interface and "1-2" and "4-5" for the left one. Out of these tracts only those highlighted in Fig. 9 b-c are used to estimate the tangential stiffness values. In particulare stage $2-3$ for the right interface and stage "1-2" for the left one are neglected due to spurious platforms' movements.

For each identified tract the central portion of the slope is singled out and fitted using a least square method. The resulting values are plotted against their respective normal load levels at the interface (see Fig. 10), together with their standard deviation.

It should be noted how the right tangential stiffness (i.e. on the cylindrical side) is not influenced by variations of the normal load, while the one on the flat side increases with increasing normal load. This may be due to the conforming nature of the flat contact interface.

\section{CONCLUSIONS}

This paper focuses on a coupled experimental-numerical approach to investigate, simulate and understand under-platform dampers behaviour. The paper highilights the strengths of this method, but also its limits concerning the estimation of friction contact parameters, with a particular emphasis on contact stiffness values.

A novel procedure and data processing technique is therefore proposed to overcome these limits and give reliable indications to the damper designer concerning the values of contact parameters to be used as input to the damper numerical routine. A sample of results is discussed in order to show, in practice, the technique to estimate the contact parameters (tangential stiffness on the cylindrical side and both tangential and normal contact stiffness on the flat interface) starting from the experimental results. Each measured quantity is assigned a level of uncertainty and the data processing technique gives an indication of the reliability of each derived quantity. These authors believe that this work constitutes a reliable basis to build a design tool (i.e. validated damper numerical routine) capable of giving trustworthy predictions in the frame of asymmetrical damper design and optimization. 


\section{REFERENCES}

[1] Gola, M. M., Liu, T. A direct experimental-numerical method for investigations of a laboratory under-platform damper behavior. International Journal of Solids and Structures, 51(25-26), pp. 4245-4259, 2014.

[2] Gastaldi, C., Gola, M. M., Understanding Complexities in Underplatform Damper Mechanics. In Proc. ASME Turbo Expo 2014, GT2014-25240, 2014.

[3] Gastaldi, C., Gola, M. M., A Random Sampling Strategy for Tuning Contact Parameters of Underplatform Dampers. In Proc. ASME Turbo Expo 2015, GT2015-42834, 2015.

[4] Schwingshackl, C.W., Petrov, E.P., and Ewins, D.J., Effects of Contact Interface Parameters on Vibration of Turbine Bladed Disks With Underplatform Dampers. Journal of Engineering for Gas Turbines and Power, 134(3), 2012.

[5] Schwingshackl, C.W., Petrov, E.P., and Ewins, D.J., Measured and estimated friction interface parameters in a nonlinear dynamic analysis. Mechanical Systems and Signal Processing, 28, pp.574-584.

[6] Gastaldi, C., Gola, M. M., Pre-optimization of Asymmetrical Underplatform Dampers. Accepted at ASME Turbo Expo 2016, GT2016-57359, 2016.

[7] Gola, M. M., Bragas Dos Santos, M., Liu, T., 2012. Measurement of the scatter of underplatform damper hysteresis cycle: experimental approach. In Proc. of ASME IDET, DETC2012-70269, pp. 359-369.

[8] Gola, M. M., Liu, T., M. Bragas Dos Santos M., Investigation of under-platform damper kinematics and its interaction with contact parameters (nominal friction coefficient)". In Proc. of World Tribology Congress, 2013.

[9] T.Harris, Rolling Bearing Analysis, 3rd Edition, John Wiley and Sons, 1991.

[10] Brändlein J., Ball and Roller Bearings : Theory, Design, and Application, John Wiley and Sons, 1999.

[11] Gola, M. M., Gastaldi, C., An improved Microslip Model for variable normal Loads, In Proc. of 9th IFToMM International Conference on Rotor Dynamics, IFToMM ICORD 2014. 